\title{
MULTI-WAVELENGTHS 3D LASER SCANNING FOR PIGMENT AND STRUCTURAL STUDIES ON THE FRESCOED CEILING "THE TRIUMPH OF DIVINE PROVIDENCE"
}

\author{
M. Guarneri ${ }^{1, *}$, S. Ceccarelli ${ }^{2}$, M. Ferri De Collibus ${ }^{1}$, M. Francucci ${ }^{1}$, M. Ciaffi ${ }^{1}$ \\ ${ }^{1}$ ENEA - R. C. Frascati, FSN-TECFIS-DIM laboratory, 00044 Frascati (RM), Italy - (massimiliano.guarneri, mario.ferridecollibus, \\ massimo.francucci, massimiliano.ciaffi)@enea.it \\ ${ }^{2}$ Dept. of Industrial Engineering, University of Rome Tor Vergata, Rome, Italy - sofia.ceccarelli@uniroma2.it
}

Commission II, WG II/8

KEY WORDS: RGB-ITR, 3D digitalization, remote colourimetry, Palazzo Barberini, LADAR, laser scanning

\begin{abstract}
:
The modern 3D digitalization techniques open new scenarios on how to transmit to the next generations the state of health of Cultural Heritage $(\mathrm{CH})$ buildings, paintings, frescos or statues. The final goal of the 3D digitalization is an exact replica of the acquired target, but a standard and unique technique able to digitalize artworks of different size and in different ambient light conditions is still far from being successfully ready for the $\mathrm{CH}$ field. Even if both laser scanning and photogrammetry can be considered mature techniques, applied with success in most of the Cultural Heritage study cases, they are limited in terms of colour digitalization and image quality in all the cases where ambient light and big sensor-target distances are crucial factors: differently to standard laser scanners, which collect colour information by the use of a coaxial camera and the distance by an IR laser source, the RGB-ITR (Red, Green and Blue Imaging Topological Radar) scanner, developed in ENEA, is equipped with three different laser sources for the simultaneous colour and distance estimation. The present work shows the results obtained applying the above-mentioned multi-wavelengths laser scanner for collecting a complete high-quality 3D colour model of "The Triumph of Divine Providence" vault, painted by Pietro da Cortona on the ceiling of the noble hall inside Palazzo Barberini in Rome.
\end{abstract}

\section{INTRODUCTION}

\subsection{Introduction and aims}

The modern 3D digitalization techniques open new scenarios on how to transmit to the next generations the state of health of Cultural Heritage $(\mathrm{CH})$ buildings, paintings, frescos or statues. The final goal of the 3D digitalization is an exact replica of the acquired target, but a standard and unique technique able to digitalize artworks of different size and in different ambient light conditions is still far from being successfully ready for the $\mathrm{CH}$ field (Pierdicca, R. et al., 2016; Remondino, F., 2011). Even if both laser scanning and photogrammetry can be considered mature techniques, applied with success in most of the Cultural Heritage study cases, they are limited in terms of colour digitalization and image quality in all the cases where ambient light and big sensor-target distances are crucial factors (Remondino, F., 2006; Callieri, M. et al., 2011).

The present work describes the adoption of a multi-wavelengths 3D laser scanner for the digitalization of the frescoed ceiling of one of the biggest rooms inside Palazzo Barberini, placed in the centre of Rome. The choice to use the multi-wavelengths scanner was dictated also by several constraints such as museum requests and room constraints: first, the area couldn't be interdicted to the visitors during the digitalization; second, any kind of scaffolds or infrastructures had to be avoided, because of their dangerousness and invasiveness; third, the illumination inside the room couldn't be altered, due to the presence of the visitors, and the staff that operated on the scanner couldn't access to the area out of the opening time of the museum. As shown in Figure 1, only an area of one squared meter surrounding the instrument was interdicted to the visitors and the scanner could operate without supervisors during the acquisition phase.

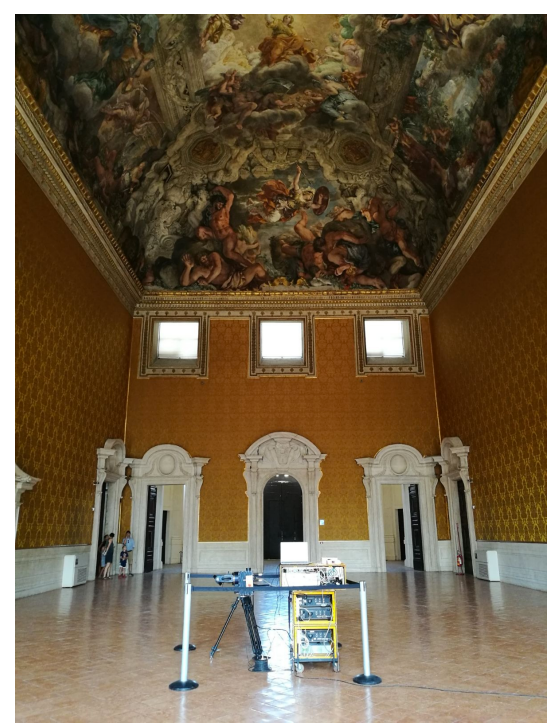

Figure 1. The RGB-ITR scanner during the digitalization inside the Pietro da Cortona's room

1.2 A brief description of the artwork: "The Triumph of Divine Providence" painting

The fresco of "The Triumph of Divine Providence" was realised by Pietro Berrettini, known also as Pietro da Cortona, on the wider room ceiling of Palazzo Barberini (Rome) for the celebration of the spiritual and political power of the Barberini

* Corresponding author 
family, accomplished with the pontificate of Maffeo, known as Urbano VIII. The painter celebrates the new Pope representing in the ceiling decoration the allegoric poem on the conflict between vices and virtues written by the poet Francesco Bracciolini for pontiff election: for this reason, the painting is often mentioned as The Allegory of Divine Providence and Barberini Power. Completed in seven years between 1632 and 1639, this monumental fresco is extended for about $400 \mathrm{~m}^{2}$ and it is considered one of the most important example of Italian Baroque painting (Briganti, 1962; Lo Bianco, 1992). The great challenge of Pietro da Cortona was for many aspects compared to the Michelangelo's masterpiece Sistine Chapel, first of all for the room's dimensions: the Barberini hall is 24 meters length, 14.5 meters width and almost 18 meters high. The artist conceived the ceiling composition as an enormous vortex of figures, architectural and natural elements, involving the spectator in an extraordinary visual and emotional adventure. The theme of The Triumph of the Divine Providence is divided in five scenes by a false monochrome frame within the rectangular area of the vault delimitated by the real cornice, representing famous episodes of Roman history alluding to civic and moral virtues, symbols of the Barberini family's values. The central scene coincides with the very core of the fresco, materializing The Triumph of Divine Providence and the accomplishment of Her aims under the pontificate of Urban VIII. Here, the Divine Providence is surrounded by many figures among which Justice, Piety, Authority, Truth, Beauty and Modesty. Upper, Immortality is depicted while supports a crown of stars, which symbolizes Urania who, following the Providence orders, crowns the entire Barberini lineage with eternal glory. The Theological virtues, Faith, Hope and Charity, support the Barberini emblem, composed by three extraordinary bees enclosed by a laurel wreath, which configures the central area of the Barberini shield. These heraldic elements are closely integrated to the ceiling decoration to the purpose of the family celebration. The figures of Rome, which raises the crown of the triregnum, and Glory with the two pontifical keys - one for divine authority and the other for the temporal one (Vitzthum, 1961) - close the central frame in the upper side. In the angular clipei, allegorical scenes are depicted with historical figures and symbolic animals, as precise references to the virtues of Barberini Pope. In the four lateral sectors, episodes that symbolize the good governance of the Barberini are painted in an allegorical and celebrative interpretation, referring to the political activity of Barberini Pope and his grandchildren.

For its importance The Triumph of Divine Providence fresco has been restored several times, from minor conservation interventions to complete restorations. The first complete restoration of the ceiling was recorded in 1971 with the aim of cleaning the surface from dirty layers and previous repainting, repairing the so-called bottacioli, rounded shape gaps generated by mortar intrinsic defects. Successively, other three restorations were performed in 1981, 1993 and 2010, providing the current aspect of the painting and the whole room (Scott, 1991; Testa, 2018).

\section{MATERIALS AND METHODS}

\subsection{The 3D acquisition and digitalization setup}

The Triumph of Divine Providence fresco was entirely digitalized by the RGB-ITR (Red Green Blue-Imaging Topological

(1) The name of the scanner was chosen by the first authors, for differentiating this technology from the existing laser scanner, which are equipped with digital cameras for colour
Radar(1)) scanner prototype, assembled and developed in the Diagnostic and Metrology Laboratory of ENEA R. C. Frascati. The system is composed by a hardware part and two software applications - one for planning the digitalization and steering the laser beam on the surface, acquiring and storing the data; the other was developed for loading the data, filtering structural information, calibrating colour texture, building and exporting the final mesh, registering data collected from different stations, analysing collected data and exporting coloured meshes for external 3D software or virtual environments.

The hardware setup is composed by two main parts: the optical head (the passive module) and the electronic components (the active module) connected together by a couple of optical fibres. This configuration has a historical reason, inherited by the laboratory expertise to develop instruments for nuclear sector: keeping the two modules separated, it is possible to place just the optical head in a radioactive environment without compromising the electronic parts.

The scanner is equipped with three different laser sources (661, 514, $440 \mathrm{~nm}$ ) combined together by a dichroic filter, which ensures both colour and structural digitisation without the use of digital camera. The colour and structural information are estimated by the lock-in amplifier technique (Stanford Research Systems, 2016; Zurich Instruments, 2018): the three lasers are amplitude-modulated by three different sinusoids; for each of the three channels the back-reflected signals are collected by three avalanche photodiodes, which convert the optical signals to the electronic ones.

The entire system is controlled by a PC laptop equipped with a custom software, called itrScansSystem, which can steer the laser beams in the desired position on the target, plan the acquisitions - dividing the digitalization in different portions with different acquisition parameters, for example the sampling frequencies, the spatial resolutions, etc - and collect the data, storing each channel in separated files. The software is able to control also a mechanical shutter, piloted by an ARDUINO ONE board, which blocks out of the optical head the propagation of the laser beams in presence of visitors, permitting to operate in safe conditions.

\subsection{ITR data resolution and accuracy}

As described before the RGB-ITR scanner is able to estimate colour and structural information by the lock-in amplifier technique.

The signals detected during the digitalization of the ceiling were in a range of $0-0.4 \mathrm{~V}$, digitally converted by a 16-bit analogue-todigital converter (ADC) with a full-scale of $20 \mathrm{~V}$. With this configuration the system was able to collect more than $2^{10}$ grey levels per each channel, ensuring a high micro-contrast in the resulting coloured textures. The spatial resolution between two points of a target placed at $17 \mathrm{~m}$ is about $0.6 \mathrm{~mm}$, equal to half of the overall lasers spot size - focused on the scene by a $40 \mathrm{~mm}$ achromatic lens - and proportional to the motors-step resolution of the scanning mirror at that distance.

In terms of dots-per-inch (dpi) the RGB-ITR is comparable with the resolution of a professional digital camera equipped with a high quality $170 \mathrm{~mm}$ lens and an APS-C sensor of $12 \mathrm{M}$ pixels. The estimation of the resolution in dpi of the RGB-ITR scanner at $17 \mathrm{~m}$ is calculated with the following equation:

$$
d p i=\frac{1 \text { inch }}{\text { half spot size }}=\frac{25.4 \mathrm{~mm}}{0.6 \mathrm{~mm}}=43 \mathrm{dpi}
$$

acquisition. It's obvious that the acronym Radar should be substituted by Lidar or Ladar, but for historical reasons the name was never changed. 
while for the camera is:

$$
d p i=\frac{|M| \cdot 1 \text { inch }}{\text { pixel size }}=\frac{0.01 \cdot 25.4 \mathrm{~mm}}{5.8 \cdot 10^{-3} \mathrm{~mm}}=44 \mathrm{dpi}
$$

where $\mathrm{M}$ represents the magnification factor, calculated by the formula:

$$
M=\frac{f}{f-D}
$$

where $\mathrm{f}$ is the focal length and $\mathrm{D}$ the distance camera-target. In the case of the digital camera we are assuming that the lenses are ideal, but in the real case the paraxial and marginal focus have to be considered, especially closed to the lens borders, causing the blur effect on the acquired image. Usually, to avoid these problems is sufficient to make smaller the camera iris aperture, increasing the depth-of-field (DoF), but reducing the quantity of light captured by the CMOS sensor. In the case of the RGB-ITR the lens diameter, $100 \mathrm{~mm}$, and the fibre optics bundle, $1 \mathrm{~mm}$, are much bigger than the image of the receiving spots size.

Another parameter, difficult to quantify, but which determines the goodness of the digitalized scene is the influence caused by external factors like the ambient light: in the case of systems based on cameras this is a crucial parameter, which can affect the final result; vice-versa, the RGB-ITR scanner collects both colours and structure information by the use of modulated laser beams that are completely independent from the environmental effects (Poujouly and Journet, 2002; Mullen et al., 2004).

\subsection{The scanner and post-processing pipelines}

The digitalization of the vault was completed in two different acquisition campaigns, especially because of some interruptions caused by some maintenance works, public events and the difficult access from the floor to part of the frescos covered by a cornice all around the perimeter of the ceiling itself. Referring to the room plant, the scanning process was completed moving the instrument in six stations as shown in the Figure 2: the first and second stations were used for digitalizing most of the entire surface and the last four for finishing the parts hidden by the cornice.

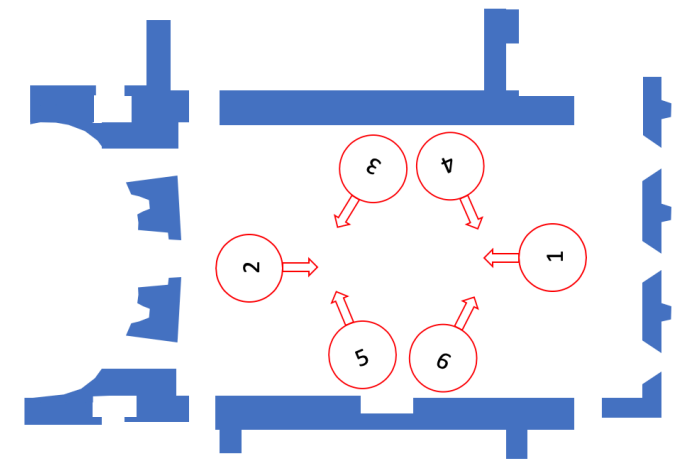

Figure 2. Plant of the Pietro da Cortona's room: the numbers from 1 to 6 represent the stations where the RGB-ITR scanner was placed and the arrows indicate the optical head directions

The first and second stations divided the scene in smaller portions to digitalize, reducing possible data loss and optical border effects: in fact, the RGB-ITR scanning-mirror drives the beams on the surface following a TV-like raster, which ensure uniform resolutions at the pole and equator sites, differently than standard monostatic LADARs that have a higher resolution at the poles.
For a better quality of both colour and structure data, the sampling frequency was set at $10 \mathrm{kHz}$ and the minimum spatial resolution at $2 \mathrm{mdeg}$. In order to minimize the vibrations effects caused by maintenance works in the room, the tripod that hosts the optical head was set at the lower height.

As described in the previous paragraph, the RGB-ITR data are not affected by environmental lights, because the scanner is based on the lock-in technique that is able to detect weak signals from a noisy environment (Zurich Instruments, 2018). In the Pietro da Cortona's room this feature was essential for permitting at the scanner to operate 24 hours per day: in fact, the presence of three big windows and an artificial light setup, hidden behind the cornice, influenced the colours used for painting the ceiling, dividing the fresco in a mosaic of sunny and shady portions.

Between the two main stations, a calibration procedure was performed for balancing and making uniform the colours during the post-processing phase: Figure 3 shows the resulting curves obtained illuminating by the lasers a white calibrated target placed at several distances from the scanner.
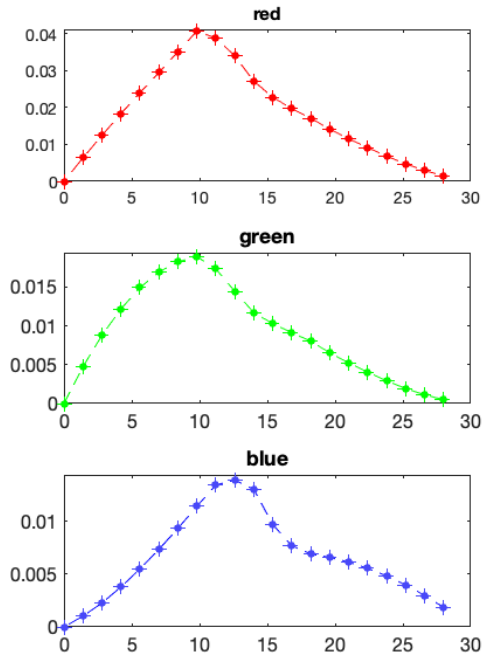

Figure 3. The calibration curves obtained in Palazzo Barberini: on the x-axis, the distances where the target was placed,

expressed in meters; on the y-axis, the amplitude per each channel of the back-reflected signal from the target, expressed in Volt

Looking at the figure, the misalignment between the signal maximum of each channels is clearly visible. This is caused by the receiving optics that focalize the three wavelengths inside the fibre optics at different distances, because the refraction index of the lens depends from the single wavelengths (Jenkins and White, 1957; Hecht, 2017).

The collected data are finally processed by a custom software, called itrAnalyzer: this software can load, calibrate, analyse and export in most of the common 3D file formats the data acquired by the scanner. All the described phases can be customized by users accessing directly to the global variables set in the memory, but for most of the purposes the internal algorithms were used. The typical post-production procedure for exporting the 3D coloured meshes and creating the entire model of the frescoed ceiling can be summarized as following: the raw structural and colour information were loaded in memory; the zig-zag effects, occurred during the digitalization phase and caused by maintenance works, were removed by applying a linear median filter with kernel dimensions of 6 rows per 1 column - this filter was necessary to correct the small misalignment visible between consecutive rows, without compromising the general quality of the images; the calibration procedure for the colour normalization, as described in (Ceccarelli et al., 2017), was 
applied; then, the colour information was transformed applying a first matrix, the elements of which are obtained by selecting on the CIE spectral curves the xyz values of each of the three wavelengths. Finally, a second matrix obtained by minimizing the colour distances of a standard coloured checkerboard measured by a MINOLTA spectrophotometer (KONICA MINOLTA, 1993; Oleari, 2008) and the RGB-ITR scanner, was applied. The matrix was found converting both scanner and spectrophotometer values in Lab colour space and minimizing their distances by the iterative closest point (ICP) algorithm (Yang and Medioni, 1991; Zhengyou, 1994). The equation (4) shows the resulting empiric matrix: looking at this matrix as a combination of affine transformations and passing to homogeneous coordinates, [L, a, b, 1] (Schneider and Eberly, 2003; House and Keyser, 2016), it is possible to notice that the two-colour coordinate systems are not only roto-translated, but also scaled.

$$
M=\begin{array}{cccc}
0.870 & -0.584 & -0.128 & -0.018 \\
-0.023 & 1.551 & 0.054 & 0.015 \\
-0.107 & -0.691 & 1.222 & 0.033 \\
0 & 0 & 0 & 1
\end{array}
$$

The equation (5) shows, in homogenous coordinates, the application of the matrix $M$ for obtaining the Lab values in the MINOLTA Spectrophotometer coordinate system from the RGB-ITR acquired data

$$
\left(\begin{array}{c}
L_{S} \\
a_{S} \\
b_{S} \\
1
\end{array}\right)=M \cdot\left(\begin{array}{c}
L_{R} \\
a_{R} \\
b_{R} \\
1
\end{array}\right)
$$

where $\mathrm{S}$ and $\mathrm{R}$ are respectively the values in the MINOLTA Spectrophotometer and RGB-ITR coordinate systems.

Also if the results obtained by the use of this scanner and presented in the next section are encouraging in terms of colour representation, further researches have to be conducted for according the RGB-ITR with the spectrophotometer values. Some of the results of the described pipeline are visible in Figure 4.

\section{RESULTS AND CONCLUSIONS}

This section will show some results obtained during the campaign for the $3 \mathrm{D}$ colour digitalization of the vault. The results are still under analysis and processing, but the 3D model can offer several starting points for further analysis and considerations about the potentiality of the technique and the collected data, useful for conservative purposes and artistic reflections.

\subsection{Results obtained after the post-processing phase}

In this paragraph all the steps used for achieving to the final 3D coloured model will be shown. In particular, the Figure 4 shows the different colour matrices obtained by several algorithms adopted for correcting the raw data resulting from the RGB-ITR digitalization: (a) is the raw colour image obtained directly from the scanner; (b) is the colour image normalized respect to the value obtained by the three calibration curves, resulting from the illumination of a calibrated white target by the three lasers and placed at several distances; (c) is the colour image obtained by the application of the matrix resulting from the CIE curves; (d) is the colour image resulting from applying a roto-translationscaling matrix, obtained empirically reducing the distances between the values acquired by the scanner and a MINOLTA spectrophotometer on a calibrated coloured checkboard; (e) shows a further image improvement through the application of algorithms for lightness amplification and of an unsharpened mask for revealing some details and enhancing the contrast of the coloured images (MOMOS Edizioni, 2018), taking advantage of the information depth of the single colour channels.

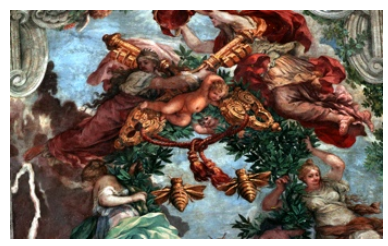

(a)

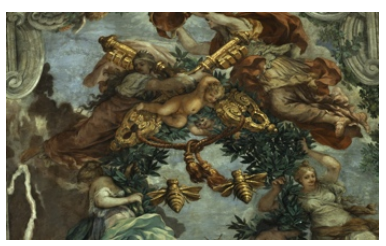

(c)

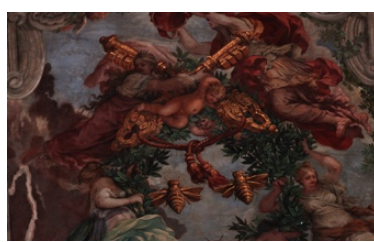

(b)

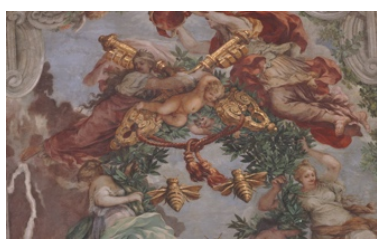

(d)

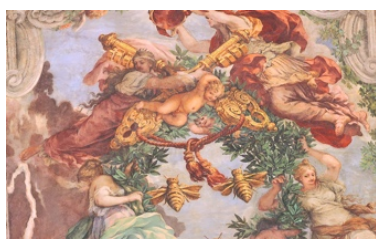

(e)

Figure 4. All the post-processing phases for arriving to the final 3D coloured model.

The Figure 5 gives a wider view of the entire digitalisation of the frescoed ceiling made by RGB-ITR scanner: looking at the figure, the uniformity of the colours can be appreciated.

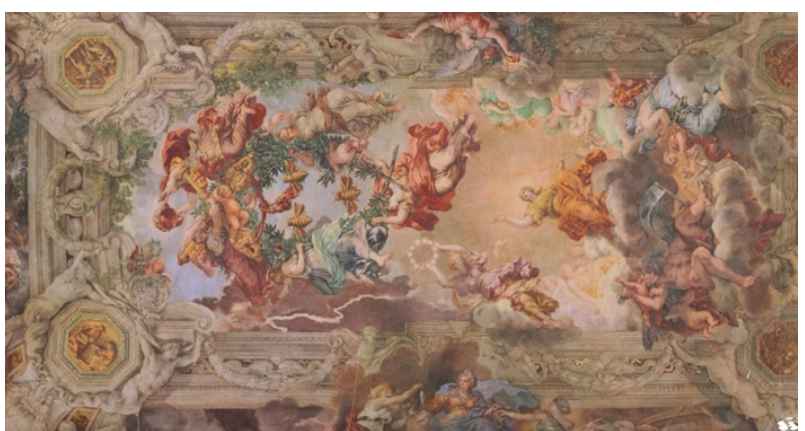

Figure 5. A view of the 3D colour model obtained by the RGBITR scanner, after all the post processing phases

Even the employed scanner cannot be considered a colorimeter, because of the limited number of wavelengths and the relative spectral information, the way how the colours are digitalized and calibrated can be used for monitoring possible modifications of the pigments of not-easily accessible artworks, like in the case of The Triumph of Divine Providence fresco. Thus the multiwavelengths 3D scanner technique can be considered a qualitative remote inspection and monitoring of the coloured and structured information of the artworks, without the need of using the scaffolds and avoiding the influence of ambient light. Moreover, any polychromatic surface digitalized by calibrated instruments, like the RGB-ITR scanner, can be inspected uniformly for each point and for all its extension, differently than the most diffused colorimeters, which can be employed for sampling just a limited number of points of the surface itself. 
An example of richness and complexity of the artwork is shown in Figure 6: the detail of the bee, emblem of the Barberini family, has dimensions $89 \times 63 \mathrm{~cm}$ with a pixel resolution of about $1497 \times 1043$. Looking at the figure is also possible to observe some restoration interventions made during the past years.

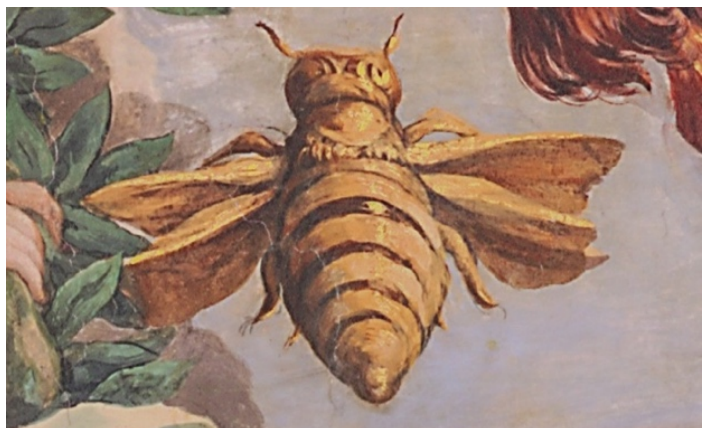

Figure 6. A detail of the fresco acquired by the scanner. The three bees were the emblem of Barberini family

As described in the previous paragraph, a median filtering with a kernel dimension of 6 rows and 1 column was applied to some portion of the digitalised surface. Figure 7 shows before (a) and after (b) the filter application. This non-linear filter is used often for removing salt-and-pepper noise, more efficiently than linear filters and with less blurring of edges (Lim, 1990). The images shown on Figure 7 were rotated of 90 degrees respect how it was stored by the instrument: in fact, the vertical misalignments visible in figure (a) are in the direction of the vertical movement of the scanning mirror, effectively corresponding to the rows of the acquired image.

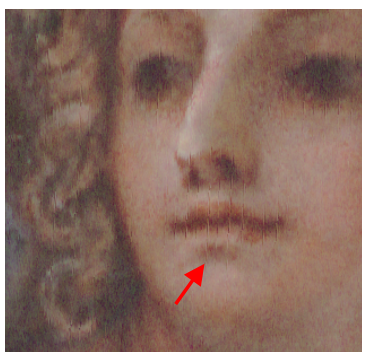

(a)

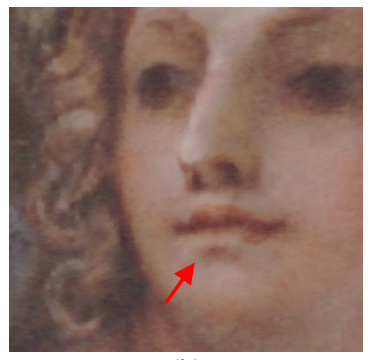

(b)
Figure 7. (a) the detail reveals several pixel misalignments caused by mechanical vibrations occurred during working maintenance works; (b) the same detail after the application of the median filter

The Figure 8 shows the differences between the images acquired by a camera and the RGB-ITR scanner, highlighting the effect caused by the sun in the first case (a) compared to the independency from the ambient light in the second one (b).

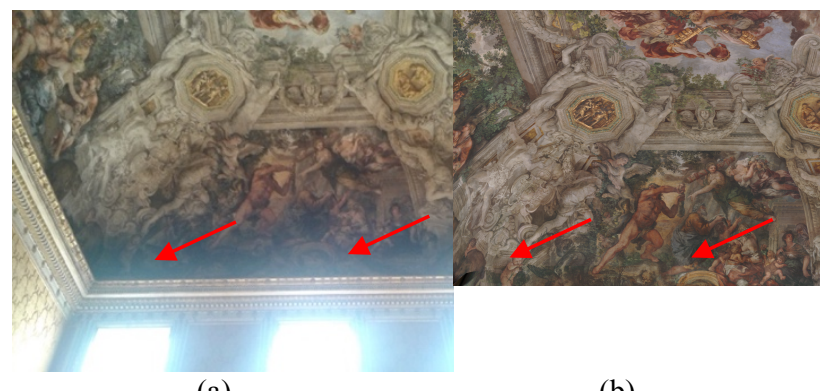

(a)

(b)

Figure 8. (a) a photo acquired by digital camera, showing the light coming inside from the windows; (b) part of the 3D model digitalized by the lasers of the RGB-ITR scanner
As described previously, the distance accuracy of the RGB-ITR scanner at $17 \mathrm{~m}$ is about $0.5-0.7 \mathrm{~mm}$ : in Figure 9 it is possible to observe the roughness of the surface. In some parts of the digitalized vault a noise contribution can be observed, especially in the areas painted with golden colours, determined by the amplitude-phase estimation effects typical of the lock-in technique in presence of no-cooperative targets: in order to reduce this effect, a calibration curve, which corrects the distance error versus target materials, has to be adopted. However, for these results the last procedure wasn't applied yet, because it is necessary to simulate the materials employed in the fresco and the increment of accuracy was not necessary for divulgation and iconographic studies of the artwork.

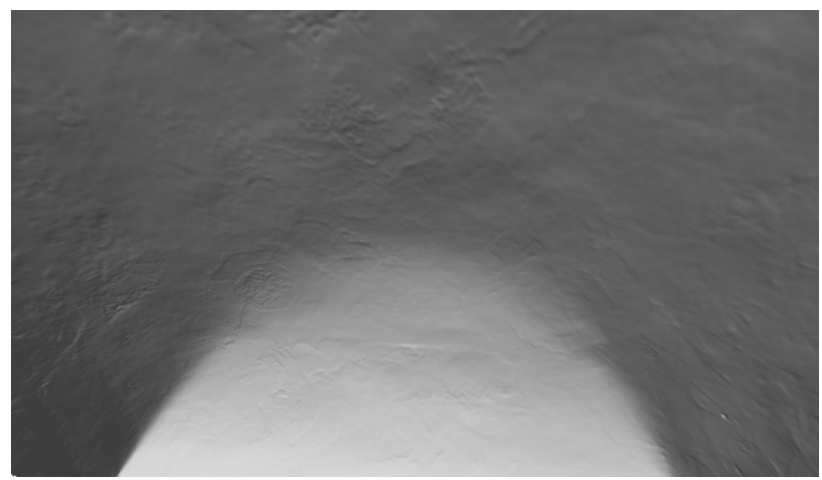

Figure 9. Part of the digitalized ceiling

\subsection{Conclusions}

The amplitude modulation of three-laser stimuli and the lock-in technique demonstrated their validity in the digitalization of The Triumph of Divine Providence fresco: the external light conditions and the characteristics of the site were a good test for the main features of the RGB-ITR laser scanner. The techniques and methodologies described demonstrated their worth and robustness especially in terms of data reusability and comparison: in fact, the intrinsic feature of the instrument to avoid the influence of ambient light, the calibration procedure for the colours normalization, the validation of the RGB-ITR data with a certified instrument, like in the case of the MINOLTA spectrophotometer, and the high accuracy of distances measurement can be used for further comparisons and investigations.

The use of this technology inside the Pietro da Cortona's room has also minimised possible data loss, which mainly could occur during the digitalisation of big surfaces. In fact, the point-to-point correlated distance and colour information open new possibilities in terms of post-production correction and image processing. For example, most of 2D image processing software have tools for the modification of pixel values by selecting a group of pixels with same intensities: the RGB-ITR scanner data can be modified using the same grouping rules, but also introducing grouping parameters based on distances selection. This feature was essential not only during the colour normalization based on calibration curves, but also for correcting intensity decreases caused by angular effects of the incident light on the surface (Ceccarelli et al., 2017).

For a full remote analysis of medium/big artworks, like that one described in the present work, and the creation of remote, 3D, multispectral, high-detailed images, more wavelengths should be introduced: this technology improvement will have also the aim to reduce the post-processing registration and interpolation phases. 
This technology, also if it is still a prototype, could be used not only in critical areas of the Cultural Heritage sector, such as archaeological sites, but also in forensic fields, such as crime scenes.

\section{ACKNOWLEDGEMENTS}

A special acknowledgement is reserved to Gallerie Nazionali Barberini Corsini, especially in charge of the Director and the staff for guesting the research group and the instruments used in the digitalization of the fresco painted by Pietro da Cortona. Another special thanks is reserved to Associazione CIVITA, which coordinated the public-private project called WeAct 3 and permitted the realization of the $3 \mathrm{D}$ model described in this work. The in-situ activities were financed by the regional project called COBRA (Sviluppo e la diffusione di metodi, tecnologie e strumenti avanzati per la COnservazione dei Beni culturali, basati sull'applicazione di Radiazioni e di tecnologie Abilitanti(2)).

\section{REFERENCES}

Briganti, G., 1962. Pietro da Cortona o della Pittura Barocca. Sansoni Editore, Firenze.

Callieri, M., Chica, A., Dellepiace, M., Besora, I., Corsini, M., Moyes, J.,Ranzuglia, G., Scopigno, R., Brunet, P., 2011. Multiscale acquisition and presentation of very large artifacts. Journal on Computing and Cultural Heritage, 3(4), pp. 1-20, doi: $10.1145 / 1957825.1957827$.

Ceccarelli, S., Guarneri, M., Fantoni, R., Giacopini, L., Danielis, A., Ferri De Collibus, M., Ciaffi, M., Fornetti, G., Francucci, M., 2017. Colorimetric Study on Optical Data from 3D Laser Scanner Prototype for Cultural Heritage Applications. In: Ceccarelli, M. and Cigola, M. (eds) New Activities For Cultural Heritage. Springer International Publishing, pp. 190-199, doi: 10.1007/978-3-319-67026-3.

Hecht, E., 2017. Optics. 5th Ed. Pearson, ISBN 9780133977226.

House, D. H. and Keyser, J. C., 2016. Foundations of Physically Based Modeling and Animation. CRC Press ISBN 9781482234602, pp. 335-341.

Jenkins, F. A., White, H., 2001. Fundamentals of Optics. 4th Ed., Fundamentals of Optics. 4th Ed. Edited by Shirley Grall. McGraw-Hill Publishing, ISBN 0072561912.

KONICA MINOLTA, 1993. Spectrophotometer CM-3500d Manual Konica Minola Sensing, Inc.

Lim, J. S., 1990. Two-Dimensional Signal and Image Processing, Englewood Cliffs, NJ, Prentice Hall, pp. 469-476.

Lo Bianco, A., 1992. Pietro da Cortona e la grande decorazione barocca. Milano.

MOMOS Edizioni, 2018. Photoshop Colori Brillanti con il metodo LAB, https://blog.momosmedia.it/photoshop-coloribrillanti-metodo-lab/.

Mullen, L., Laux, A., Concannon, B., Zege, E.P., Katsev I. L., Prikhach, A. S., 2004. Amplitude-modulated laser imager. Applied Optics, 43(19), p. 3874. doi: 10.1364/AO.43.003874.

Oleari, C., 2008. Misurare il Colore. Edited by U. Hoepli. Milano.

Pierdicca, R., Frontoni, E., Malinverni, E. S., Colosi, F., Orazi, R., 2016. Virtual reconstruction of archaeological heritage using

(2) Development and dissemination methods, technologies and advanced tools for the conservation of Cultural Heritage, a combination of photogrammetric techniques: Huaca Arco Iris, Chan Chan, Peru. Digital Applications in Archaeology and Cultural Heritage, 3(3). doi: 10.1016/j.daach.2016.06.002.

Poujouly, S., Journet, B., 2002. A twofold modulation frequency laser range finder. Journal of Optics A: Pure and Applied Optics, 4(6), pp. S356-S363. doi: 10.1088/1464-4258/4/6/380.

Remondino, F., 2006. Image-based 3D modelling: a review, The Photogrammetric Record, 21(115), pp. 269-291.

Remondino, F., 2011. Heritage recording and 3D modeling with photogrammetry and 3D scanning, Remote Sensing, 3(6), pp. 1104-1138. doi: 10.3390/rs3061104.

Schneider, P. K., Eberly, D. H., 2003. Geometric Tools for Computer Graphics. Morgan Kaufmann. p. 98. ISBN 978-155860-594-7.

Scott, J. B., 1991. Images of Nepotism. The Painted Ceilings of Palazzo Barberini, Princeton, Princeton University Press.

Stanford Research Systems, 2016. SR844 Lock-In Amplifier. Sunnyvale, California: Stanford Research Systems, Inc. Available at: https://www.zhinst.com/sites/default/files/ziUHF_UserManual_ 42300.pdf

Testa, M., 2018. Pietro da Cortona, Il Trionfo della Divina Provvidenza e il compiersi dei suoi fini sotto il pontificato di Urbano VIII Barberini.

Vitzthum, W., 1961. A Comment on the Iconograpy of Pietro da Cortona's Barberini Ceiling, The Burlington Magazine. Vol. 103, No. 703, pp. 426-431+433, http://www.jstor.org/stable/873383.

Yang, C., Medioni, G., 1991. Object modelling by registration of multiple range images. Image Vision Comput. 10 (3): 145-155. doi:10.1016/0262-8856(92)90066-C

Zhengyou, Z., 1994. Iterative point matching for registration of free-form curves and surfaces. International Journal of Computer Vision. 13 (12): 119-152. CiteSeerX 10.1.1.175.770. doi:10.1007/BF01427149

Zurich Instruments, 2018. UHF User Manual. Available at: https://www.zhinst.com/sites/default/files/ziUHF_UserManual_ 42300.pdf

based on the application of radiation and Enabling Technologies 